\title{
Seite Eins
}

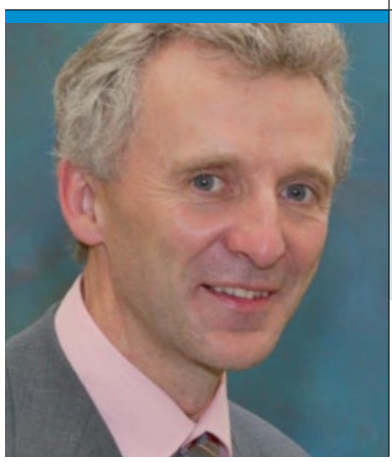

\section{Fundstücke}

Dr. med. Martin Claßen, Chefredakteur

\section{Zusammenspiel: Epilepsie und Allergie}

Eine Verbindung zwischen Störungen des ZNS und Nahrungsmittelallergien wurde schon früher postuliert, z. B. bei Migräne und ADHS. In einer Studie aus Rom wurde nun nach Einflüssen von Kuhmilchallergien auf die Rolando-Epilepsie untersucht [Lucarelli S et al. Arch Dis Child 2012 Jan 31 (Epub ahead of print)]. Bei 20 von 56 getesteten Kinder zwischen vier und 14 Jahren mit RolandoEpilepsie fiel der Pricktest positiv aus. Bei diesen Kindern führte eine einmonatige kuhmilchfreie Diät zum Verschwinden der neurologischen Symptome und zur Normalisierung des EEG. Nach erneuter Nahrungsbelastung mit Kuhmilchprotein traten bei 17 von 20 Patienten die Anfälle wieder auf. Auch wenn die Ergebnisse zunächst sehr erstaunen, scheint es lohnenswert, diesen Zusammenhang weiter zu untersuchen.

\section{Zuversicht hilft bei Schreibabys}

Auch erfahrene Kliniker verstehen oft nicht, warum sich das Schreiverhalten von Säuglingen so stark unterscheidet. Den Einfluss von pränatalem Stress und der Selbstwirksamkeitserwartung von Müttern untersuchte nun eine Arbeitsgruppe aus Basel bei 120 Schwangeren [Bolten Ml et al. J Pediatr 2012 Jan 28 (Epub ahead of print)]. Die Selbstwirksamkeitserwartung wird definiert als der Glaube einer Person an seine eigene Fähigkeit, persönliche Ziele zu erreichen. Es zeigte sich, dass die Selbstwirksamkeitserwartung der Mütter einen modulierenden Effekt auf die Dauer von Unruhe und Schrei-

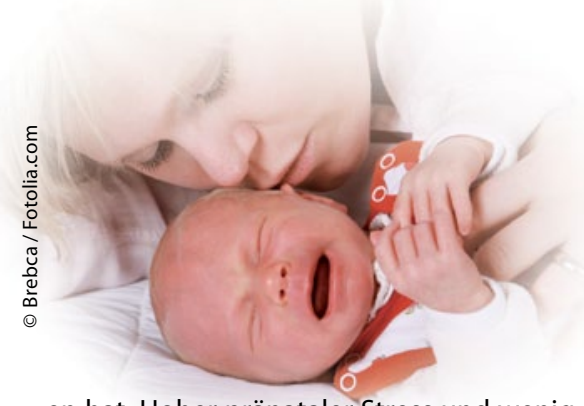

en hat: Hoher pränataler Stress und wenig Glaube an die eigenen Fähigkeiten führten zu einer deutlichen Verstärkung der Symptome bei den Kindern. Die Studie zeigt, dass maternale Faktoren einen erheblichen Einfluss auf die Entwicklung zu einem Schreibaby haben.

\section{Sexualkundeunterricht und Schwangerschaft}

Dass rigide Erziehungspraktiken und ein religiöses Umfeld nicht vor Teenagerschwangerschaften schützen, wurde verschiedentlich schon vermutet. Die USA hat mit Teenagerschwangerschaften ein besonderes Problem: Die Geburtenrate bei 15-19-Jährigen liegt mit 42,5/1.000 doppelt so hoch wie in Großbritannien und zehnmal so hoch wie in der Schweiz. Ob dies durch eine Sexualerziehung in der Schule zu verbessern wäre, untersuchten Forscher aus den USA [Cavazos-Rehg PA et al. Arch Pediatr Adolesc Med 2012; 166: 134-40]: Ein landesweiter Vergleich zeigte eine niedrigere Rate von Teenagerschwangerschaften in den Bundestaaten mit Sexualerziehung im Lehrplan. Wenn man aber als weitere Variable die demographischen, politischen und religiösen Charak-

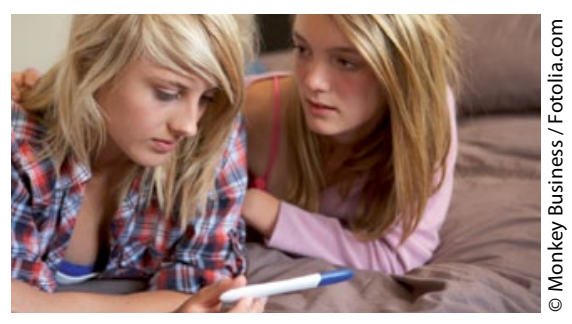

teristika und die Abtreibungspolitik berücksichtigte, nivellierte sich dieser Effekt. Insgesamt hatten die Bundesstaaten mit höherem Religiositäts-Ranking und konservativerer Regierung höhere Geburtenraten unter Jugendlichen. Die Ergebnisse zeigen, dass Wissensvermittlung alleine nicht ausreicht. Angesichts der gesellschaftspolitischen Wetterlage werden sich die anderen Faktoren aber vorerst nicht ändern.

\section{Alkoholprävention per Facebook}

Auklärungsprogramme zur Suchtprävention bei Adoleszenten erreichen häufig ihre Zielgruppe nicht. Die Möglichkeiten, die dabei soziale Netzwerke wie

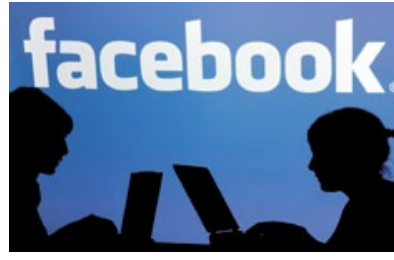

Facebook bieten, wurden von einer Arbeitsgruppe aus Washington bei 18-20-jährigen Universitätsstudenten untersucht [Moreno MA et al. Arch Pediatr Adolesc Med 2012; 166: 157-63]. Durch Analyse der Profile der Nutzer konnten diese in drei Gruppen eingeteilt werden: solche ohne öffentliche Hinweise auf Alkoholgebrauch, solche mit Hinweisen auf Alkoholgebrauch ohne Missbrauch $(19,6 \%)$ und solche, die per Bild oder Text auf Alkoholintoxikationen oder Abhängigkeit hindeuteten (16,1\%). Bei 224 Studenten konnte zusätzlich in einem Interview ein etabliertes Screening-Tool für problematischen Alkoholkonsum (AUDIT) angewendet werden. Dabei hatten diejenigen, die bei Facebook ",auffällig" waren, auch nach AUDIT eine höhere Wahrscheinlichkeit (OR 4,4) eines Alkoholproblems und eine signifikant höhere Rate alkoholbedingter Verletzungen. Der Ansatz, durch Facebook auch die richtige Zielgruppe für Interventionen zu erreichen, scheint erfolgversprechend - die Möglichkeiten der neuen Medien sollten genutzt werden. 